\title{
Phenotype in parkinsonian and nonparkinsonian LRRK2 G2019S mutation carriers
}

C. Marras, MD, PhD

B. Schuele, MD

R.P. Munhoz, MD

E. Rogaeva, $\mathrm{PhD}$

J.W. Langston, MD

M. Kasten, MD

C. Meaney, MSc

C. Klein, MD

P.M. Wadia, MD

S.-Y. Lim, MD

R.S.-I. Chuang, MD

C. Zadikof, MD

T. Steeves, MD

K.M. Prakash, MD

R.M.A. de Bie, MD

G. Adeli, MD

T. Thomsen, MD

K.K. Johansen, MD

H.A. Teive, MD

A. Asante, MSc

W. Reginold

A.E. Lang, MD

Address correspondence and reprint requests to Dr. Connie Marras, 399 Bathurst St.,

Toronto, ON M5T 2S8 Canada cmarras@uhnresearch.ca

Editorial, page 310

See page 319

Supplemental data at www.neurology.org

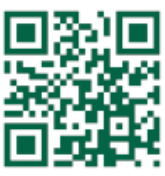

Scan this code with your smartphone to access this feature

\section{ABSTRACT}

Objectives: Using a family study design, we describe the motor and nonmotor phenotype in probands with LRRK2 G2019S mutations and family members and compare these individuals to patients with idiopathic Parkinson disease (iPD) and unrelated controls.

Methods: Probands with G2019S mutations and their first-degree relatives, subjects with iPD, and unrelated control subjects were identified from 4 movement disorders centers. All underwent neurologic examinations and tests of olfaction, color vision, anxiety, and depression inventories.

Results: Tremor was more often a presenting feature among 25 individuals with LRRK2associated PD than among 84 individuals with iPD. Subjects with LRRK2-PD had better olfactory identification compared with subjects with iPD, higher Beck Depression Inventory scores, and higher error scores on Farnsworth-Munsell 100-Hue test of color discrimination. Postural or action tremor was more common among 29 nonmanifesting mutation carriers compared with 53 noncarriers within the families. Nonparkinsonian family members had higher Unified Parkinson's Disease Rating Scale motor scores, more constipation, and worse color discrimination than controls, regardless of mutation status.

Conclusions: Although tremor is a more common presenting feature of LRRK2-PD than IPD and some nonmotor features differed in degree, the phenotype is largely overlapping. Postural or action tremor may represent an early sign. Longitudinal evaluation of a large sample of nonmanifesting carriers will be required to describe any premotor phenotype that may allow early diagnosis. Neurology ${ }^{\circledR} 2011 ; 77: 325-333$

\section{GLOSSARY}

100-Hue test $=$ Farnsworth-Munsell 100-Hue test for color discrimination; B-SIT = Brief Smell Identification Test; CASI = Cognitive Abilities Screening Instrument; iPD = idiopathic Parkinson disease; MDS-UPDRS = Movement Disorder Societysponsored revision of the Unified Parkinson's Disease Rating Scale; PD = Parkinson disease; MMSE = Mini-Mental State Examination; STAI = State-Trait Anxiety Inventory; UPDRS = Unified Parkinson's Disease Rating Scale.

Mutations in the LRRK2 gene are responsible for approximately $5 \%$ of familial and $1 \%-2 \%$ of sporadic Parkinson disease (PD) in most European populations studied. The phenotype of LRRK2-associated PD has been described as indistinguishable from idiopathic PD (iPD) with minor exceptions. ${ }^{1}$ The similarities include both motor and nonmotor phenomena. Estimates of penetrance vary widely but the penetrance of the most common G2019S mutation is clearly incomplete. ${ }^{2}$ Currently, it is not possible to predict who among unaffected carriers of mutations will develop PD in the future. There is increasing evidence that the earliest abnormalities in PD are outside the motor system. ${ }^{3}$ Studying premotor signs during life is hampered by an inability to identify individuals prior to the development of parkinsonism. The discovery of LRRK2 mutations provides an opportunity to study premotor PD and gain insight into the

From the Toronto Western Hospital and the Edmond J. Safra Program in Parkinson's Disease (C. Marras, C. Meaney, P.M.W., S.-Y.L., R.S.-I.C., C.Z., T.S., K.M.P., R.M.A.d.B., G.A., T.T., K.K.J., A.A., W.R., A.E.L.) and Centre for Research in Neurodegenerative Diseases (E.R.), University of Toronto, Toronto, Canada; The Parkinson's Institute (B.S., J.W.L.), Sunnyvale, CA; Movement Disorders Unit (R.P.M., H.A.T.), Neurology Service, Federal University of Paraná, Curitiba, PR, Brazil; Departments of Psychiatry (M.K.) and Neurogenetics (C.K.), University of Lübeck, Lübeck, Germany; and Norwegian University of Science and Technology (K.K.J.), Trondheim, Norway.

Study funding: Supported by the National Parkinson Foundation, the Parkinson Study Group, the Parkinson Disease Foundation, the Edmund J. Safra Philanthropic Association, EU grant GENEPARK (EU-LSHB-CT-2006-037544), and the NGFNplus (BMBF PNP- 01GS08135-3). C.K. is a recipient of a career development award from the Volkswagen Foundation and from the Hermann and Lilly Schilling Foundation. C.M. is a recipient of a New Investigator Award from the Canadian Institutes of Health Research.

Disclosure: Author disclosures are provided at the end of the article. 
spectrum of neurologic abnormalities that precede parkinsonism. By assessing nonmotor signs that have been associated with iPD we may be able to identify those at highest risk. This would not only be important for counseling but also for future testing and use of neuroprotective interventions. Currently, little is known about nonmotor features in LRRK2 mutation carriers without parkinsonism. We undertook a detailed evaluation of probands and family members with LRRK2 G2019S mutations in order to describe the motor and nonmotor phenotype and to compare these individuals to patients with iPD and unrelated controls.

METHODS Recruitment. Probands with LRRK2 G2019S mutations from movement disorders clinics in 3 centers (Toronto Western Hospital Movement Disorders Centre in Toronto, Canada; The Parkinson's Institute in Sunnyvale, CA; and the State of Parana Parkinson's Association in Curitiba, Brazil) were identified through genetic testing of clinic patients with PD who had a family history or age at onset less than 50. Probands with $L R R K 2$ mutations from the fourth participating center (Lübeck, Germany) were identified through unselected screening of consecutive patients with PD. For comparison purposes, patients with idiopathic PD (iPD) meeting Queen Square Brain Bank criteria for PD and individuals without neurologic disease who do not belong to LRRK2 families were recruited from movement disorders clinics in Kiel and Hamburg, Germany, and from community neurologists near these cities. Nonblood relatives of the patients with iPD and volunteers identified through advertisement were recruited by the German centers as controls without neurologic disease (unaffected controls). No preset sample size was set for the groups.

Genetic analysis. The presence of the G2019S mutation was evaluated using a restriction digest assay ( $\mathrm{SfcI}$ ) as described previously ${ }^{4}$ for the Toronto, Curitiba, and Sunnyvale subjects and using direct sequencing for the German subjects. Controls were also tested for mutations. Probands were also examined for mutations in Parkin, PINK1, [alpha]-synuclein, GBA, and DJ-1 (Toronto, Curitiba, and Sunnyvale subjects only for $G B A$ and DJ-1) genes, but none were found.

Evaluations. Probands were asked to participate in comprehensive neurologic evaluations, and to invite their first-degree relatives to participate. Any affected family member was also asked to send a letter of invitation to their first-degree relatives. In-person evaluations were performed between 2006 and 2009 and included a neurologic examination and videotape, the Unified Parkinson's Disease Rating Scale (UPDRS), the State-Trait Anxiety Inventory (STAI), the Beck Depression Inventory, the Brief Smell Identification Test (B-SIT), the Farnsworth-Munsell 100-Hue test for color discrimination (100-Hue test), and the Cognitive Abilities Screening Instrument (CASI). ${ }^{5}$ A Mini-Mental State Examination (MMSE) score can be estimated from the CASI.

Assessment of parkinsonism. For all LRRK2 probands and relatives, the presence of parkinsonism was assessed blind to the mutation status by the in-person examiner and independently by videotape review (C.M. or A.E.L.). When the 2 evaluations disagreed on any feature, a second blinded independent video review by a third neurologist was undertaken and the majority opinion was used. Parkinsonism was defined as present when at least 2 of rest tremor, bradykinesia, rigidity, or postural instability were present. Rigidity was accepted from the in-person examiner alone. If one or more of the 2 or 3 features identified was rated only as questionably present, then a rating of "questionable parkinsonism” was assigned. Subjects with definite or questionable parkinsonism were evaluated for the presence of PD by 2 expert reviewers (C.M. and A.E.L.) using all available information (medical history, medical records, video, in-person examiner's ratings). The most likely diagnosis was determined according to 1) Queen Square Brain Bank Criteria (modified to allow a family history of $\mathrm{PD}$ ) and 2) clinical expert opinion. Individuals with parkinsonism and $L R R K 2$ mutations were designated as manifesting carriers and any signs atypical for PD were noted. Subjects with iPD and unaffected controls also underwent a clinical evaluation including the UPDRS by a neurologist to confirm the presence or absence of parkinsonism.

Standard protocol approvals, registrations, and patient consents. All subjects provided written informed consent to participate. The study was approved by the ethics board of the University Health Network, Toronto, Canada, and that of each individual participating site.

Statistical analysis. Manifesting carriers were divided into 4 groups defined by disease duration and phenotypic features were summarized within each group. Disease duration was calculated as the interval between symptom onset and evaluation. Demographic, motor, and nonmotor features in individuals with LRRK2-PD and iPD were compared. Relevant symptoms and signs were also compared among nonparkinsonian mutation carriers, noncarrier relatives, and unrelated controls. Comparisons were repeated in the subset of nonparkinsonian individuals over 45 years of age. Continuous variables were compared using linear regression adjusting for age and (for PD group comparisons) disease duration. Categorical variables were compared using logistic regression adjusting for the same covariates. No adjustment was made for multiple comparisons. To address differences in age and disease duration between the LRRK2 and iPD groups, 3 sets of sensitivity analyses were performed: 1) excluding outlying observations from the LRRK2-PD group that did not overlap with values in the $\mathrm{PPD}$ group, 2) excluding the youngest subjects with iPD, and 3) excluding the subjects with iPD with the shortest disease duration.

German subjects were evaluated using the old UPDRS while subjects from all other centers were evaluated using the Movement Disorder Society-sponsored revision of the UPDRS (MDS-UPDRS). ${ }^{6}$ To facilitate comparison, we modified the motor MDS-UPDRS to retain only compatible items between old and new UPDRS. The items freezing (3.11) and rest tremor constancy (3.18) were removed. To rate leg bradykinesia, the highest score on items 3.7 (toe tapping) and 3.8 (leg agility) for each leg were taken as the score for that leg. To rate postural or kinetic tremor, the highest score on items 3.15 (postural tremor) and 3.16 (kinetic tremor) for each arm was taken as the score for that arm. No imputation was made for missing data. Subjects missing values on a particular outcome were not included in the analysis for that particular outcome.

RESULTS Fifteen unrelated probands with G2019S mutations were recruited ( 4 from Toronto, 2 from Bra- 


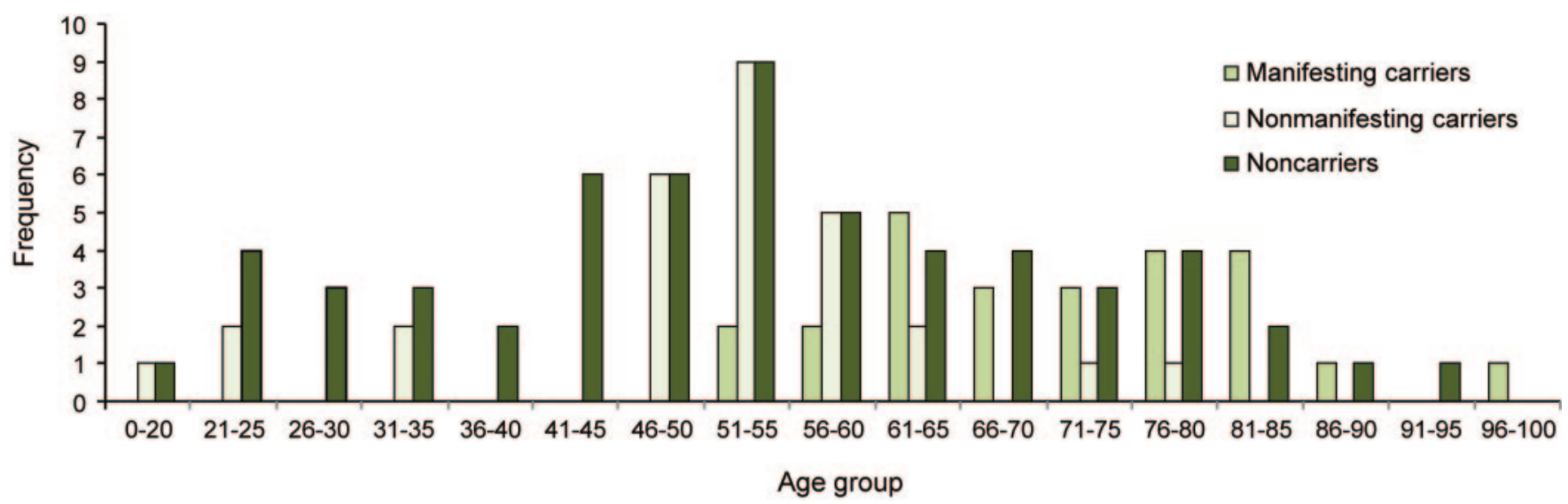

Age at the time of evaluations for all LRRK2 family members, grouped by clinical status presence or absence of parkinsonism (manifesting or nonmanifesting) and mutation status.

zil, 6 from California, and 3 from Germany). None declined to participate. A total of 112 relatives were examined from the 15 families. Two subjects did not provide blood samples; however, the related parent for both of these individuals was tested and was negative for the mutation. The subjects were therefore considered negative for the mutation. However, the married-in spouse was not tested for the mutation.

LRRK2 mutation carriers. Among the 112 examined subjects, 54 carriers of LRRK2 G2019S mutations were identified. The ages at examination of the $L R R K 2$ family member subjects are shown in figure 1. Twenty-five mutation carriers were determined to have parkinsonism ( 9 from Toronto, 7 from California, 6 from Brazil, and 3 from Germany). Twentyfour met Queen Square Brain Bank Criteria for PD (modified to allow a family history of PD). Past medical history on the remaining subject was not sufficiently detailed to apply the criteria. All were believed by expert opinion to have a most likely diagnosis of PD. Neurologic diagnoses in the 29 nonmanifesting carriers were cervical dystonia and questionable parkinsonism (1), possible cervical dystonia (1), craniopharyngioma (1), and chronic inflammatory demyelinating polyneuropathy (1). Five relatives without mutations were determined to have parkinsonism with the following clinical diagnoses: PD (1), progressive supranuclear palsy (1), and parkinsonism with dementia not further defined (3). Other neurologic diagnoses in noncarrier family members were tics (1) and segmental dystonia (1).

The clinical characteristics of the LRRK2 manifesting carriers are shown in tables 1 and 2. Tremor was the most commonly recognized initial symptom. A total of 23 of 24 manifesting carriers who had been treated with dopaminergic medication reported a good response. All subjects were examined in the "on" state except one subject who was not treated with dopaminergic medication. Table 2 shows the clinical features divided in groups according to disease duration at examination. Postural instability $(6 / 8)$ and gait disorders (7/8) were common even in early disease. Motor fluctuations and dyskinesias were common. Olfactory identification was abnormal for age in only 2 of the 7 tested individuals with short disease duration ( $0-5$ years) but was abnormal in $8 / 10$ with very long duration ( $\geq 16$ years). Other nonmotor features of PD (constipation, urinary dysfunction, sleep disturbance, depression, and anxiety) were common from early disease and did not become more prevalent in the longer disease duration groups. Color discrimination was abnormal for age (as de-

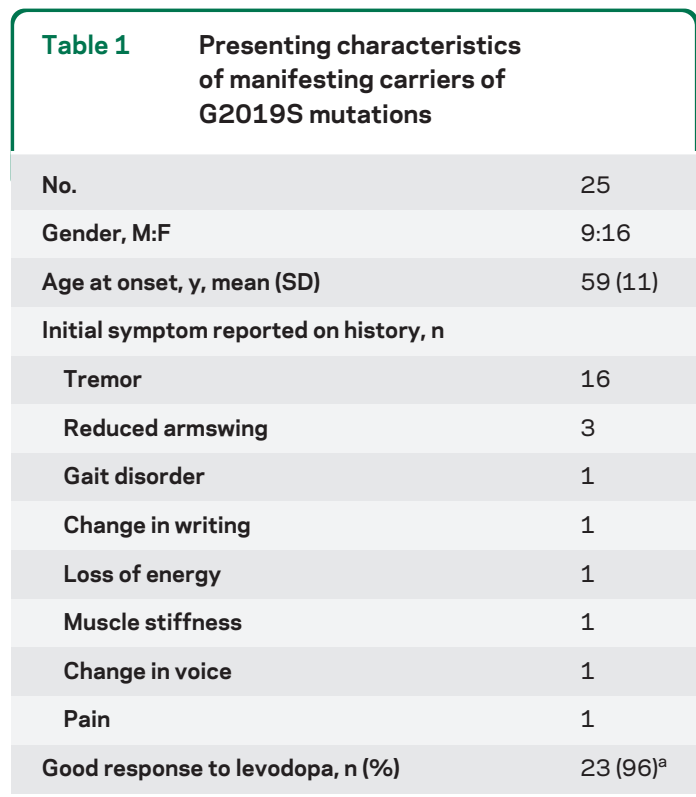

a One subject was untreated. 
Table 2 Characteristics of manifesting carriers by disease duration at examination ${ }^{a}$

\begin{tabular}{|c|c|c|c|c|c|}
\hline & $0-5$ years & $6-10$ years & $11-15$ years & $16+$ years & All \\
\hline No. & 8 & 2 & 5 & 10 & 25 \\
\hline Age at evaluation, $y$ & $63(10)$ & $71(11)$ & $76(12)$ & $78(9)$ & $72(11)$ \\
\hline \multicolumn{6}{|l|}{ UPDRS } \\
\hline Motor score $(\max 108)^{b}$ & $21(12)$ & $19(9)$ & $28(13)$ & $21(10)$ & $22(11)$ \\
\hline Nonmotor EDL ( $\max 42)$ & $8(5)(m=2)$ & $2(2)$ & $7(5)(m=1)$ & $7(5)$ & $7(5)(m=3)$ \\
\hline Motor EDL (max 42) & $12(7)$ & $4(0)$ & $16(13)(m=1)$ & $15(10)$ & $13(9)(m=1)$ \\
\hline Rest tremor, n (\%) & $6(75)$ & $1(50)$ & $2(40)$ & $6(60)$ & $15(60)$ \\
\hline Gait disorder, n (\%) & $7(88)$ & $2(100)$ & $5(100)$ & $7(70)$ & $21(84)$ \\
\hline Postural instability, $\mathrm{n}(\%)$ & $6(75)$ & $1(50)$ & $4(80)$ & $8(80)$ & $19(76)$ \\
\hline \multicolumn{6}{|l|}{$\%$ of day in "off " state $(m=2)$} \\
\hline 0 & 6 & 2 & 1 & 2 & 11 \\
\hline $1-25$ & 2 & 0 & 4 & 3 & 10 \\
\hline $25-50$ & 0 & 0 & 0 & 0 & 1 \\
\hline $50-75$ & 0 & 0 & 0 & 2 & 0 \\
\hline $75-100$ & 0 & 0 & 0 & 1 & 1 \\
\hline \multicolumn{6}{|l|}{$\%$ of day with dyskinesias $(m=2)$} \\
\hline 0 & 7 & 2 & 1 & 2 & 12 \\
\hline $1-25$ & 1 & 0 & 4 & 3 & 8 \\
\hline $25-50$ & 0 & 0 & 0 & 0 & 0 \\
\hline $50-75$ & 0 & 0 & 0 & 2 & 2 \\
\hline $75-100$ & 0 & 0 & 0 & 1 & 1 \\
\hline MMSE score & $29(1)(m=4)$ & $28(1)$ & $29(2)(m=1)$ & $23(10)(m=2)$ & $26(7)(m=7)$ \\
\hline Constipation, n (\%) & $3(38)$ & $1(50)$ & $3(60)$ & $4(40)$ & $11(44)$ \\
\hline Current or prior depression, $\mathrm{n}(\%)$ & $6(75)$ & $0(0)$ & $1(20)$ & $4(50)(m=2)$ & $11(48)(m=2)$ \\
\hline Beck Depression Inventory score & $20(21)(m=4)$ & $3(4)$ & $11(6)(m=1)$ & $10(8)(m=4)$ & $12(12)(m=9)$ \\
\hline Current or prior symptoms of anxiety, $n(\%)$ & $3(38)$ & 0 & 0 & $1(13)(m=2)$ & $4(17)(m=2)$ \\
\hline STAI-S score (missing = 10) & $48(16)(m=4)$ & $24(m=1)$ & $34(9)(m=1)$ & $40(9)(m=2)$ & $40(9)(m=8)$ \\
\hline B-SIT percentile score, mean (SD) & $36(29)(m=1)$ & $61(0)$ & $29(26)$ & $27(22)$ & $33(25)(m=1)$ \\
\hline Normal:abnormal for age & $5: 2$ & $2: 0$ & $4: 1$ & $2: 8$ & $13: 11$ \\
\hline $100-$ Hue test error score (mean, SD) & $183(108)$ & $192(6)$ & $234(131)$ & $186(37)(m=2)$ & $194(84)(m=2)$ \\
\hline Errors above 95 th percentile for age, $n(\%)$ & $4(50)$ & $2(100)$ & $3(75)(m=1)$ & $1(13)(m=2)$ & $10(45)(m=3)$ \\
\hline
\end{tabular}

Abbreviations: 100-Hue test = Farnsworth-Munsell 100-Hue test for color discrimination; B-SIT = Brief Smell Identification Test; $E D L=$ experiences of daily living; $m$ = missing; MDS-UPDRS = Movement Disorder Society-sponsored revision of the Unified Parkinson's Disease Rating Scale; MMSE = Mini-Mental State Examination; STAI-S = State-Trait Anxiety Inventory State score; UPDRS = Unified Parkinson's Disease Rating Scale.

a All values are mean (SD) unless otherwise noted.

b UPDRS motor subscale is from the original UPDRS for German subjects and from MDS-UPDRS for all others. The motor MDS-UPDRS was modified to retain only compatible items between old and new UPDRS. From the MDS-UPDRS the following were removed: freezing (3.11) and rest tremor constancy (3.18). For leg bradykinesia: for each leg the highest score on items 3.7 (toe tapping) and 3.8 (leg agility) were taken as the score for that leg. For postural/kinetic tremor: for each arm the highest score on items 3.15 (postural tremor) and 3.16 (kinetic tremor) was taken as the score for that arm.

fined by an error score above the 95th percentile for age $^{7}$ ) in 11/19 tested individuals.

LRRK2-PD compared with iPD. Table 3 compares the clinical features of 84 subjects with iPD to those with LRRK2-PD. Subjects with iPD were younger and had shorter disease duration at the time of examination than those with LRRK2-PD. The overlap of both age and disease duration is substantial (figures e-1 and e-2 on the Neurology ${ }^{\circledR}$ Web site at www. neurology.org) and statistical adjustment was deemed a valid approach. All comparisons are adjusted for age and disease duration. Tremor was more common as an initial symptom in LRRK2-PD and gait dysfunction was more frequent at the time of evaluation, adjusting for disease duration. There were no significant differences in UPDRS scores between the groups, or in the 


\begin{tabular}{|c|c|c|c|}
\hline \multirow{2}{*}{ Comparison of LRRI } & ted $\mathrm{PD}$ and idiopathic $\mathrm{PD}$ & & \multirow[b]{2}{*}{$p$ Value } \\
\hline & LRRK2-PD ( $\mathrm{n}=25)$ & Idiopathic PD ( $\mathrm{n}=84)$ & \\
\hline Age at evaluation, $y$, mean (SD) & $72(11)$ & $63(10)$ & 0.001 \\
\hline Disease duration, $y$, mean (SD) & $12(9)$ & $6(6)(m=6)$ & 0.001 \\
\hline Gender, M:F & $12: 13$ & 49:35 & 0.36 \\
\hline Age at onset, $y$, mean (SD) & $59(11)$ & $54(14)(m=6)$ & 0.15 \\
\hline Levodopa equivalent dose, mg & 631 (410) & $585(423)(m=11)$ & 0.69 \\
\hline Good response to levodopa, $n(\%)$ & $23(96)^{b}$ & $65(83)^{b}(m=6)$ & 0.52 \\
\hline \multicolumn{4}{|c|}{ Initial symptom reported on history, $\mathrm{n}(\%)$} \\
\hline Tremor & $16(64)$ & 31 (39) & $0.02^{\mathrm{c}}$ \\
\hline Gait disorder & $1(4)$ & $11(14)$ & \\
\hline Muscle stiffness/rigidity & $1(4)$ & $5(6)$ & \\
\hline Reduced armswing & $3(13)$ & 2 (3) & \\
\hline Change in writing & $1(4)$ & $3(4)$ & \\
\hline Loss of energy & $1(4)$ & $4(5)$ & \\
\hline Loss of dexterity & 0 & $14(18)$ & \\
\hline Other & $2(8)$ & $9(11)$ & \\
\hline Missing & 0 & 5 & \\
\hline \multicolumn{4}{|l|}{$\begin{array}{l}\text { The following are adjusted for age and } \\
\text { disease duration (see footnote) }\end{array}$} \\
\hline $\begin{array}{l}\text { UPDRS motor subscale score } \\
\text { (maximum 108) }^{d}\end{array}$ & $20.1(15.2-25.0)$ & $25.8(23.2-28.4)(m=6)$ & 0.05 \\
\hline Rest tremor & $0.66(0.42-0.86)(m=1)$ & $0.49(0.37-0.61)(m=6)$ & 0.19 \\
\hline Gait disorder & $0.84(0.55-0.96)(m=1)$ & $0.54(0.41-0.67)(m=7)$ & 0.03 \\
\hline Postural instability by pull test & $0.73(0.43-0.91)(m=1)$ & $0.63(0.50-0.75)(m=6)$ & 0.46 \\
\hline Motor fluctuation & $0.43(0.19-0.70)(m=2)$ & $0.34(0.21-0.50)(m=9)$ & 0.49 \\
\hline Dyskinesias & $0.23(0.05-0.52)(m=2)$ & $0.32(0.19-0.50)(m=8)$ & 0.51 \\
\hline Constipation & $0.40(0.17-0.64)(m=2)$ & $0.41(0.29-0.53)(m=11)$ & 0.94 \\
\hline $\begin{array}{l}\text { Current or prior symptoms of } \\
\text { depression }\end{array}$ & $0.50(0.24-0.73)(m=2)$ & $0.27(0.17-0.39)(m=8)$ & 0.07 \\
\hline Beck Depression Inventory score & $12.6(8.9-16.3)(m=9)$ & $8.3(6.6-9.9)(m=13)$ & 0.04 \\
\hline STAI-S score & $42.3(36.3-48.3)(m=10)$ & $38.9(36.2-41.6)(m=23)$ & 0.33 \\
\hline B-SIT percentile score & $34.6(25.9-43.3)(m=1)$ & $13.3(8.7-17.9)(m=10)$ & $<0.0001$ \\
\hline $\begin{array}{l}\text { Farnsworth-Munsell 100-Hue test } \\
\text { error score }\end{array}$ & $184(147-221)(m=3)$ & $127(109-146)(m=6)$ & 0.01 \\
\hline Errors above $95^{\text {th }}$ percentile for age & $0.51(0.26-0.75)(m=3)$ & $0.25(0.16-0.37)(m=6)$ & 0.05 \\
\hline
\end{tabular}

Abbreviations: 100-Hue test = Farnsworth-Munsell 100-Hue test for color discrimination; B-SIT = Brief Smell Identification Test; iPD = idiopathic Parkinson disease; $\mathrm{m}$ = missing; MDS = Movement Disorder Society; PD = Parkinson disease; STAI-S = State-Trait Anxiety Inventory State score; UPDRS = Unified Parkinson's disease Rating Scale.

${ }^{a}$ Least square means (95\% confidence intervals) are reported for continuous variables adjusting for age and disease duration using analysis of covariance. For categorical variables, predicted probabilities are reported adjusting for age and disease duration \pm gender using multiple logistic regression.

b One LRRK2 mutation carrier and 9 subjects with iPD had not been tried on dopaminergic medication.

${ }^{\mathrm{c}}$ For tremor vs all other initial symptoms.

${ }^{\mathrm{d}}$ Modified to merge old and new (MDS) UPDRS scores. See text for details.

frequency of complications of dopaminergic therapy. $L R R K 2$ and iPD subjects with $\geq 16$ years of disease duration had very similar UPDRS motor scores $(30.2 \pm$ 13.2 vs $29.7 \pm 10.6$ ).

Several differences were observed among nonmotor symptoms. LRRK2-PD subjects had better olfactory identification compared with iPD subjects; however, they had higher Beck Depression Inventory scores and higher error scores (worse performance) on the 100-Hue test of color discrimination.

When the analyses were repeated excluding 8 outlying LRRK2-PD subjects with the oldest age or longest disease duration or reducing the iPD group to the oldest (mean age $70 \pm 7$ years) or longest duration (mean duration $10 \pm 6$ years), we found the same results (tables e- 1 through e-3). 


\begin{tabular}{|c|c|c|c|c|}
\hline \multirow[t]{2}{*}{ Table 4} & d psychiatric features i & nonparkinsonian muta & on carriers vs noncarriers & \multirow[b]{2}{*}{$p$ Value } \\
\hline & $\begin{array}{l}\text { (a) G2019S mutation } \\
\text { carrier }(n=29)\end{array}$ & $\begin{array}{l}\text { (b) Noncarriers from } \\
\text { LRRK2 families ( } n=53 \text { ) }\end{array}$ & $\begin{array}{l}\text { (c) Unrelated controls } \\
(\mathrm{n}=112)\end{array}$ & \\
\hline Age & $50.5( \pm 13.8)$ & $51.1( \pm 17.0)$ & $60.1( \pm 10.5)$ & $<0.0001$ \\
\hline Gender, M:F & $16: 13$ & $26: 27$ & $61: 51$ & 0.79 \\
\hline UPDRS motor score ${ }^{+}$ & $2.4(1.4-3.4)$ & $2.2(1.4-2.9)$ & $0.7(0.2-1.2)(m=3)$ & 0.001 \\
\hline Postural/action tremor & $0.31(0.15-0.53)$ & $0.12(0.04-0.25)$ & $0.13(0.08-0.21)(m=4)$ & $\begin{array}{l}0.04^{a-c} \\
0.82^{b-c^{\prime}} \\
0.05^{a-b^{\prime}}\end{array}$ \\
\hline Constipation & $0.12(0.03-0.32)(m=1)$ & $0.14(0.06-0.28)(m=2)$ & $0.02(0.00-0.06)(m=12)$ & $\begin{array}{l}0.04^{a-c} \\
0.008^{b^{\prime}-c} \\
0.78^{a-b}\end{array}$ \\
\hline B-SIT percentile score & 39 (29-49) & $30(23-40)(m=1)$ & $40(35-45)(m=3)$ & 0.13 \\
\hline $100-$ Hue test error score & $173(143-202)(m=1)$ & $136(114-158)(m=3)$ & $95(80-109)$ & $\begin{array}{l}<0.0001^{\ddagger} \\
0.11^{\mathrm{a}-\mathrm{b}}\end{array}$ \\
\hline $\begin{array}{l}\text { Errors above 95th } \\
\text { percentile for age, } n(\%)\end{array}$ & $0.48(0.28-0.67)(m=2)$ & $0.46(0.32-0.60)(m=3)$ & $0.15(0.09-0.23)$ & $\begin{array}{l}0.0001^{b-c} \\
0.01^{a-c}\end{array}$ \\
\hline Prior or current depression & $0.28(0.13-0.48)$ & $0.33(0.21-0.48)(m=1)$ & $0.27(0.19-0.37)(m=6)$ & NS \\
\hline $\begin{array}{l}\text { Beck Depression Inventory } \\
\text { score }\end{array}$ & $5.6(3.3-7.9)(m=1)$ & $6.3(4.6-8.0)(m=4)$ & $5.2(4.0-6.4)(m=13)$ & 0.6041 \\
\hline STAI-S & $30.2(26.5-33.9)(m=1)$ & $32.6(29.8-35.4)(m=4)$ & $33.8(31.7-35.9)(m=23)$ & 0.27 \\
\hline
\end{tabular}

Abbreviations: 100-Hue test $=$ Farnsworth-Munsell 100-Hue test for color discrimination; B-SIT = Brief Smell Identification Test; $\mathrm{m}$ = missing; MDS = Movement Disorder Society; STAI-S = State-Trait Anxiety Inventory State score; UPDRS = Unified Parkinson's disease Rating Scale.

* Least square means (95\% confidence intervals) are reported for continuous variables adjusting for age using analysis of covariance. For categorical variables, predicted probabilities are reported adjusting for age \pm gender using multiple logistic regression.

${ }^{+}$Modified to merge old and new (MDS) UPDRS scores. See text for details.

₹ Post hoc test comparisons: 0.2279 , $^{\text {a-b }}<0.00011^{a-c} 0.0123 .^{b-c}$

Nonmanifesting G2019S mutation carriers compared with noncarriers. Table 4 shows neurologic and psychiatric features in 29 nonmanifesting G2019S mutation carriers, 53 related noncarriers without parkinsonism, and 112 unrelated controls. The nonmanifesting mutation carriers were a similar age to their noncarrier relatives but were younger than the unrelated controls. The nonmanifesting carriers were on average 9 years younger at the time of examination than the mean age at onset of the manifesting carriers. All analyses are adjusted for age. The greatest differences were between the unaffected controls and the family members, regardless of the presence of the mutation in the family members. Motor UPDRS scores were higher in the family members than in unrelated controls. Family members more frequently reported constipation and had higher 100-Hue test error scores (worse color discrimination). The only notable difference between unaffected mutation carriers and noncarriers within the families was a marginally significant difference in the frequency of postural and action tremor (more common in mutation carriers). When the 3 groups without parkinsonism were restricted to those over the age of 45 , this difference became more marked $(38 \%$ vs $10 \%, p=0.01)$. Otherwise, the results were the unchanged by the restricted age range, which brought the groups much closer together in average age (table e-4).

Eleven percent $(3 / 29)$ of nonmanifesting mutation carriers and 21\% (11/53) of nonparkinsonian related noncarriers were believed to have one or more parkinsonian signs that together did not meet criteria for parkinsonism. One mutation carrier (age 57) and one related noncarrier (age 25) were believed to have questionable parkinsonism by global impression that did not meet diagnostic criteria for PD.

DISCUSSION Our findings are in agreement with the majority of reports of the clinical features of LRRK2-associated PD, which indicate that the phenotype is not distinguishable from iPD. There have been individual exceptions to this, however; atypical cases with supranuclear gaze palsies, ${ }^{8}$ prominent dementia, ${ }^{9}$ or psychosis unrelated to medications. ${ }^{10}$ Tremor was a more common presenting feature in our LRRK2 group compared with iPD, and this was also found in a large international study. ${ }^{1}$ It has been suggested that the course of LRRK2-PD is more benign than that of $\mathrm{iPD}$, when compared to cases from a brain tissue bank. ${ }^{1}$ In our direct comparison, individuals with $\mathrm{PPD}$ and LRRK2-PD of long duration had similar UPDRS motor scores suggesting a similar course. In contrast, a comparison of 73 Tunisian 
subjects with PD with LRRK2 G2019S mutation with 107 subjects with PD without known genetic cause found higher UPDRS motor scores and more dyskinesias in the mutation carriers after adjusting for disease duration and age. ${ }^{11}$ Longitudinal evaluation would more convincingly define the course of LRRK2-PD.

The nonmotor features found in iPD were also found in our LRRK2-PD cases, but some differences in degree were noted. Better (though abnormal) olfactory function in LRRK2-PD compared with iPD is in keeping with suggestions from several other groups. ${ }^{1,12,13}$ Yet other groups have found frequencies of hyposmia similar to iPD. ${ }^{14,15}$ To our knowledge, comparisons of color vision, depressive symptoms, or anxiety have not previously been made directly with iPD, although depression and anxiety have been noted to be common. ${ }^{1,14,16}$

This is the largest group of nonmanifesting carriers described to date, to our knowledge. The only difference we found between nonmanifesting mutation carriers and related noncarriers was a higher frequency of postural or action tremor in the mutation carriers. The specificity of this as a predictor of future parkinsonism is likely to be low, as it was also present in $13 \%$ of the unrelated controls. Previous studies of asymptomatic LRRK2 mutation carriers have reported subtle gait changes detected by accelerometer. ${ }^{17}$ Another study found no differences on MMSE, Mattis Dementia Rating Scale, other cognitive tests, or olfactory function between 12 nonmanifesting carriers aged 40 to 73 and 8 related noncarriers. ${ }^{18}$ In another study, 2 asymptomatic carriers were found to have normal olfaction at the ages of 36 and $47 . .^{15}$

Among the subjects without parkinsonism, it was notable that the major differences were found between the unrelated controls and the family members, regardless of mutation status. Higher motor UPDRS scores in the family members may be due to greater vigilance for these signs on the part of the examiners, who were aware of the related (though not the mutation) status of the family members, or volunteer bias, if family members with subtle symptoms were more interested to participate. Other differences between unaffected family members and controls (e.g., color discrimination, rates of constipation) that are less susceptible to examiner bias suggest the clustering of neurologic signs within these families. If real, it suggests that the relatives have subclinical motor and nonmotor signs (and perhaps, by extension, a predisposition to PD) that are independent of LRRK2 mutations. Most of the nonmanifesting family members examined belong to multiplex families, and some coexisting risk factors, either environmental or genetic, could account for high pen- etrance as well as the presence of 4 individuals with parkinsonism in the absence of a LRRK2 mutation (phenocopies) in these families. The high prevalence of dystonia (3 individuals of 112 examined, 2 of whom had LRRK2 mutations) is also notable and further suggests predisposing factors to neurologic dysfunction in these families.

Two important features of our sample are relevant to the interpretation of the results. First, volunteer biases may accentuate differences between nonmanifesting relatives and unrelated controls, due to different motivations for participating. Second, many comparisons were performed, increasing the possibility of significant findings due to chance alone. Although our sample is the largest group of nonmanifesting carriers reported to date, our sample is still small, limiting our ability to identify statistical significance for any but large effects. Our findings need to be confirmed in other studies to assess their reproducibility.

iPD is clinically heterogeneous. It may encompass several diseases with an overlapping spectrum of manifestations. ${ }^{19,20}$ Our data and those of others suggest that LRRK2-PD fits well within this clinical spectrum, with a tendency to be more homogeneous in some respects than all of iPD (e.g., tremordominant, uniformly good response to levodopa). If this is so, LRRK2 mutations provide an important mechanism for identifying an at-risk cohort, and a model for studying pathophysiologic changes present at the earliest stages of PD as well as interventions for disease prevention or delaying disease onset or progression. Premotor abnormalities are subtle if present and longitudinal evaluations in unselected LRRK2 mutation cohorts will be necessary to document whether or not and when nonmanifesting carriers develop nonmotor neurologic abnormalities and whether or not these are antecedents to parkinsonism.

\section{AUTHOR CONTRIBUTIONS}

Dr. Marras: drafting/revising the manuscript, study concept or design, analysis or interpretation of data, acquisition of data, statistical analysis, study supervision, obtaining funding. Dr. Schuele: drafting/revising the manuscript, study concept or design, acquisition of data, study supervision. Dr. Munhoz: drafting/revising the manuscript, acquisition of data. Dr. Rogaeva: analysis or interpretation of data, obtaining funding. Dr. Langston: drafting/revising the manuscript, study concept or design, analysis or interpretation of data, acquisition of data, study supervision, obtaining funding. Dr. Kasten: drafting/revising the manuscript, analysis or interpretation of data, acquisition of data. C. Meaney: drafting/revising the manuscript, analysis or interpretation of data, statistical analysis. Dr. Klein: drafting/revising the manuscript, analysis or interpretation of data, acquisition of data, obtaining funding. Dr. Wadia: drafting/revising the manuscript, acquisition of data. Dr. Lim: drafting/revising the manuscript, acquisition of data. Dr. Chuang: drafting/revising the manuscript, acquisition of data. Dr. Zadikoff: drafting/revising the manuscript, acquisition of data. Dr. Steeves: drafting/revising the manuscript, analysis or interpretation of data, acquisition of data. Dr. Prakash: drafting/revising the manuscript, acquisition of data. Dr. de Bie: drafting/revising the manuscript, acquisition of data. Dr. Adeli: study concept or design, contribu- 
tion of vital reagents/tools/patients. Dr. Thomsen: drafting/revising the manuscript, acquisition of data. Dr. Johansen: drafting/revising the manuscript, contribution of vital reagents/tools/patients. Dr. Teive: drafting/ revising the manuscript, analysis or interpretation of data, acquisition of data. A. Asante: analysis or interpretation of data, acquisition of data, statistical analysis, study supervision. W. Reginold: analysis or interpretation of data. Dr. Lang: study concept or design, analysis or interpretation of data, acquisition of data, study supervision, obtaining funding.

\section{ACKNOWLEDGMENT}

The authors thank Francis Baraquio, Patricia Williams, Hannah Reay, Dorotea Mutabdzic, Manoj Enjati, and Vera Tadic for assistance with project and data management; Dr. Rosane Nisenbaum for assistance with statistical analysis; and Dr. Joyce van der Vegt and Dr. Carsten Buhmann for examination of the control group subjects.

\section{DISCLOSURE}

Dr. Marras has served as a consultant for Solvay Pharmaceuticals, Inc.; and has received research support from Merck Serono, the National Parkinson Foundation, Parkinson Study Group, Parkinson Disease Foundation, Michael J Fox Foundation, and the Canadian Institutes of Health Research. Dr. Schuele receives research support from the California Institute for Regenerative Medicine, the Blume Foundation, and the Parkinson's Alliance. Dr. Munhoz reports no disclosures. Dr. Rogaeva receives research support from the Canadian Institutes of Health Research and the Ontario Research Fund. Dr. Langston serves on scientific advisory boards for Teva Pharmaceutical Industries Ltd., Merck Serono, NeuroNova, the Michael J Fox Foundation, and Elan Corporation; has received speaker honoraria from Teva Pharmaceutical Industries Ltd., Parkinson's Resources of Oregon, and Northwest Parkinson's; is listed as an author on patents re: Use of nicotine to treat L-dopa dyskinesias, High-throughput screening for anti-aggregation small molecules to block alpha-synuclein aggregation, Electrophysiological approach using heart rate variability to screen for pre-motor PD, and Use of agents that block GBA activity to treat PD; serves as a consultant for Elan Corporation, Teva Pharmaceutical Industries Ltd., and Lundbeck Inc.; serves on the speakers' bureau for Teva Pharmaceutical Industries Ltd.; receives royalties from sales of a psychometric testing tool (Cognistat), and has received research support from the US Department of Defense, the NIH/NIEHS, California Institute for Regenerative Medicine, the Michael J Fox Foundation, and The Blume Foundation/Stanford University. Dr. Kasten has received funding for travel from the Melvin Yahr Foundation and receives research support from the German Research Foundation (DFG). C. Meaney reports no disclosures. Dr. Klein has received speaker honoraria from GlaxoSmithKline, Boehringer Ingelheim, Merz Pharmaceuticals, LLC, and Orion Corporation; serves on the editorial boards of Neurology ${ }^{\circledR}$, Movement Disorders, and Parkinson's Disease; serves as a consultant for Centogene, Boehringer Ingelheim, and Link Medicine; and receives research support from the German Research Foundation (DFG), intramural funding from the University of Lübeck, the Volkswagen Foundation, the Possehl Foundation, the Hermann and Lilly Schilling Foundation, and the Fritz Thyssen Foundation. Dr. Wadia reports no disclosures. Dr. Lim has received speaker honoraria from Boehringer Ingelheim, GlaxoSmithKline, Novartis, and Roche; serves as an Associate Editor for Neurology Asia; serves as a consultant for GlaxoSmithKline and Novartis; and has received research support from the University of Malaya. Dr. Chuang reports no disclosures. Dr. Zadikof has served on speakers' bureaus for and received speaker honoraria from Allergan, Inc., Teva Pharmaceutical Industries Ltd., Lundbeck Inc., and Ipsen; receives research support from Solvay Pharmaceuticals, Inc., the Michael J Fox Foundation, the Dystonia Medical Research Foundation, the National Parkinson Foundation, and the $\mathrm{NIH}$; and has served as an expert witness in a medico-legal case. Dr. Steeves and Dr. Prakash report no disclosures. Dr. de Bie has received funding for travel from Medtronic, Inc. and Abbott; and has received research support from Medtronic, Inc., ZonMw Doelmatigheid, Het Internationaal Parkinson Fonds, Parkinson Vereniging, and Het Prinses Beatrix Fonds. Dr. Adeli reports no disclosures. Dr. Thomsen received unrestricted fellowship funding from Boehringer Ingelheim. Dr. Johansen reports no disclosures. Dr. Teive serves on the editorial boards of Parkin- sonism and Related Disorders and Arquivos de Neuro-Psiquiatria. A. Asante and W. Reginold report no disclosures. Dr. Lang has served on scientific advisory boards for Abbott, Allon Therapeutics, Inc., Biovail Corporation, Boehringer Ingelheim, Cephalon, Inc., Ceregene, Eisai Inc., Medtronic, Inc. Lundbeck Inc., NeuroMolecular Pharmaceuticals, Novartis, Merck Serono, Solvay Pharmaceuticals, Inc., TaroPharma, and Teva Pharmaceutical Industries Ltd.; has received speaker honoraria from GlaxoSmithKline and UCB; receives/has received research support from the Canadian Institutes of Health Research, the Dystonia Medical Research Foundation, the Michael J Fox Foundation, the National Parkinson Foundation, and the Ontario Problem Gambling Research Centre; and has served as an expert witness in cases related to the welding industry.

Received August 25, 2010. Accepted in final form January 11, 2011.

\section{REFERENCES}

1. Healy DG, Falchi M, O'Sullivan SS, et al. Phenotype, genotype, and worldwide genetic penetrance of LRRK2associated Parkinson's disease: a case-control study. Lancet Neurol 2008;7:583-590.

2. Latourelle JC, Sun M, Lew MF, et al. The Gly2019Ser mutation in LRRK2 is not fully penetrant in familial Parkinson's disease: the GenePD study. BMC Med 2008;6:32.

3. Braak H, Del Tredici K, Rub U, et al. Staging of brain pathology related to sporadic Parkinson's disease. Neurobiol Aging 2003;24:197-211.

4. Paisan-Ruiz C, Lang AE, Kawarai T, et al. LRRK2 mutation in Parkinson's disease: mutation analysis and casecontrol association study. Neurology 2005;65:696-700.

5. Teng EL, Hasegawa K, Homma A, et al. The Cognitive Abilities Screening Instrument (CASI): a practical test for cross-cultural epidemiological studies of dementia. Int Psychogeriatr 1994;6:45-58.

6. Goetz CG, Tilley BC, Shaftman SR, et al. Movement Disorder Society-sponsored revision of the Unified Parkinson's Disease Rating Scale (MDS-UPDRS): scale presentation and clinimetric testing results. Mov Disord 2008;23:2129-2170.

7. Kinnear PR, Sahraie A. New Farnsworth-Munsell 100 hue test norms of normal observers for each year of age 5-22 and for age decades 30-70. Br J Ophthalmol 2002;86: $1408-1411$.

8. Zimprich A, Biskup S, Leitner P, et al. Mutations in LRRK2 cause autosomal-dominant parkinsonism with pleomorphic pathology [see comment]. Neuron 2004;44: 601-607.

9. Santos-Reboucas CB, Abdalla CB, Baldi FJ, et al. Cooccurrence of sporadic parkinsonism and late-onset Alzheimer's disease in a Brazilian male with the LRRK2 p.G2019S mutation. Genet Test 2008;12:471-473.

10. Tomiyama H, Li Y, Funayama M, et al. Clinicogenetic study of mutations in LRRK2 exon 41 in Parkinson's disease patients from 18 countries. Mov Disord 2006;21: 1102-1108.

11. Nishioka K, Kefi M, Jasinska-Myga B, et al. A comparative study of LRRK2, PINK1 and genetically undefined familial Parkinson's disease. J Neurol Neurosurg Psychiatry 2010;81:391-395.

12. Hernandez DG, Paisan-Ruiz C, McInerney-Leo A, et al. Clinical and positron emission tomography of Parkinson's disease caused by LRRK2. Ann Neurol 2005;57:453-456.

13. Valldeoriola F, Gaig C, Muxi A, et al. 123I-MIBG cardiac uptake and smell identification in patients with LRRK2 mutations. Mov Disord 2010;25:S298. 
14. Ferreira JJ, Guedes LC, Rosa MM, et al. High prevalence of $L R R K 2$ mutations in familial and sporadic Parkinson's disease in Portugal. Mov Disord 2007;22: 1194-1201.

15. Silveira-Moriyama L, Guedes LC, Kingsbury A, et al. Hyposmia in G2019S LRRK2-related parkinsonism: clinical and pathologic data. Neurology 2008;71:10211026.

16. Goldwurm S, Zini M, Di Fonzo A, et al. LRRK2 G2019S mutation and Parkinson's disease: a clinical, neuropsychological and neuropsychiatric study in a large Italian sample. Parkinsonism Relat Disord 2006;12:410-419.
17. Mirelman A, Gurevich T, Wseiss A, et al. Gait in health carriers and non-carriers of the LRRK2 G2019S mutation: preliminary evidence of subtle preclinical motor changes. Mov Disord 2010;25:294.

18. Lohmann E, Leclere L, De Anna F, et al. A clinical, neuropsychological and olfactory evaluation of a large family with LRRK2 mutations. Parkinsonism Relat Disord 2009;15:273-276.

19. Weiner WJ. There is no Parkinson disease. Arch Neurol 2008;65:705-708.

20. Marras C, Lang AE. Changing concepts in Parkinson's disease: beyond the decade of the brain. Neurology 2008;70: 1996-2003.

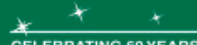 CELEBRATING 60 YEARS
OF PUBLICATION $++\star$

CLINICAL DIAGNOSIS OF ALZHEIMER'S DISEASE: REPORT OF THE NINCDS-ADRDA WORK GROUP UNDER THE AUSPICES OF DEPARTMENT OF HEALTH AND HUMAN SERVICES TASK FORCE ON ALZHEIMER'S DISEASE

Guy McKhann, MD; David Drachman, MD; Marshall Folstein, MD; Robert Katzman, MD; Donald Price, MD; Emanuel M. Stadlan, MD

Neurology 1984;34:939-944

Clinical criteria for the diagnosis of Alzheimer's disease include insidious onset and progressive impairment of memory and other cognitive functions. There are no motor, sensory, or coordination deficits early in the disease. The diagnosis cannot be determined by laboratory tests. These tests are important primarily in identifying other possible causes of dementia that must be excluded before the diagnosis of Alzheimer's disease may be made with confidence. Neuropsychological tests provide confirmatory evidence of the diagnosis of dementia and help to assess the course and response to therapy. The criteria proposed are intended to serve as a guide for the diagnosis of probable, possible, and definite Alzheimer's disease; these criteria will be revised as more definitive information becomes available.

Free Access to this article at www.neurology.org/content/34/7/939

Comment from David S. Knopman, MD, FAAN, Deputy Editor: This highly cited paper presents diagnostic criteria, but these have been foundational for the field. The NINCDS-ADRDA criteria for AD dementia have stood up over the last 27 years-they are still fundamentally valid and well-framed. 